\title{
Incidence of nonalcoholic fatty liver diseases and their associated risk factors among the type-2 diabetic population
}

\author{
Usha Sree Puneem ${ }^{1,3}$, Subburaya Mudaliyar Rajendran Ramakrishnan², Sindgi Vasudeva Murthy³, Vanitha Rani \\ Nagasubramanian ${ }^{1 *}$ \\ ${ }^{1}$ Department of Pharmacy Practice, Sri Ramachandra Institute of Higher Education Research, Deemed to be University, Chennai, India. \\ ${ }^{2}$ Department of General Medicine, Sri Ramachandra Institute of Higher Education and Research Deemed to be University, Chennai, India. \\ ${ }^{3}$ Department of Pharmacy Practice, Jayamukhi College of Pharmacy, Narasampet, India.
}

\section{ARTICLE INFO \\ Received on: 26/03/2021 \\ Accepted on: 29/05/2021 \\ Available online: 05/07/2021}

\section{Key words:}

Nonalcoholic fatty liver

disease, type-2 diabetes

mellitus, South India.

\begin{abstract}
The aim of the study was to investigate the incidence and factors linked with nonalcoholic fatty liver disease (NAFLD) among type-2 diabetes mellitus (T2DM) patients in Warangal, Telangana, India. It was an observational, cohort crosssectional study; a target sample of 100 T2DM patients was recruited from a single study site of Warangal. Detailed medical history was obtained and laboratory examinations and ultrasound imaging were carried out to evaluate the NAFLD. The overall incidence of NAFLD was $80 \%$. The risk of NAFLD was significantly high in subjects with uncontrolled blood glucose levels, abnormal waist circumference, increased aspartate aminotransferase (AST), and elevated triglyceride levels $(p<0.05)$. In our study subjects, the consumption of alcohol is the predominant risk factor (Odds ratio: 6.652; 95\% confident interval: $1.86-23.74$ and $p=0.00 *$ ) significantly causing NAFLD among the study subjects. Grade- 1 and 2 fatty livers are typically associated with elevated AST levels and a high incidence was observed in men.
\end{abstract}

\section{INTRODUCTION}

Nonalcoholic fatty liver disease (NAFLD) manifests in all genders, ages, and ethnic groups. The principle hazardous factors of NAFLD are insulin resistance, type-2 diabetes mellitus (T2DM), hyperlipidemia, and obesity. NAFLD is most prevalent globally with the incidence of $25 \%$ and $29.32 \%$ in Asian continent countries (Kim et al., 2018). The annual incidence of NAFLD was 51 cases per 1000 people, which may progress to chronic fibrosis, cirrhosis, and hepatic cancer (Goh et al., 2016; Li et al., 2019). Based on the lifestyle, the incidence of NAFLD varies from rural to urban. It is low among the rural population (9\%) and is high among the urban population ranging from $16 \%$ to $32 \%$ (Singh et al., 2004).

The prevalence of NAFLD is greater among T2DM patients (Vernon et al., 2011). NAFLD is characterized by insulin resistance and compensatory hyperinsulinemia, leading to faulty

"Corresponding Author

Vanitha Rani Nagasubramanian, Department of Pharmacy Practice, Sri Ramachandra Institute of Higher Education Research, Deemed to be University, Chennai, India.E-mail: vanitharani.n@sriramachandra.edu.in lipid metabolism, hepatic accumulation of triglycerides, or dysfunction of beta-cells in T2DM patients (Forlani et al., 2016). Globally, the implication of NAFLD is 55.5\% and nonalcoholic steatohepatitis is $37.3 \%$ among type-2 diabetic patients (Younossi et al., 2019). Evidence shows that NAFLD-T2DM affiliation is bidirectional (Li et al., 2017).

Data pertaining to type-2 diabetic patients with NAFLD and without NAFLD are lacking in Telangana. Hence, keeping this as an objective in mind, we quantified the liver function tests and measured the grades of fatty liver among the screened subjects to assess the incidence and factors associated with hepatic steatosis (fatty liver) and advanced liver fibrosis among the type-2 diabetic patients in Telangana, South India.

\section{MATERIALS AND METHODS}

\section{Study design, setting, and population}

The study was a cross-sectional, single center study, where the target patients attended the Department of Gastroenterology Clinic, Warangal, from January 2020 to January 2021. Willing participants of both genders, with an age range of 20-65 years with a history of T2DM were included and patients with a history 
of alcoholic liver disease and history of other chronic liver disease, such as hepatitis, were excluded from the study.

\section{Sample size and sampling}

The sample size was calculated based on a previous study conducted in South India (Mohan et al., 2009), assuming $95 \%$ confidence interval and $6 \%$ absolute precision with an estimate of $32 \%$ since no prior estimate from Warangal. Hence, we were ascertained to include 100 subjects, and those who attended the gastroenterology clinic, Warangal, were included based on the study protocol.

\section{Anthropometric indices and biochemical analysis}

A standardized protocol comprising anthropometric parameters such body mass index (BMI) and waist circumference (WC) were measured and calculated. BMI was calculated as body weight $(\mathrm{kg})$ divided by height $\left(\mathrm{m}^{2}\right)$ and the subjects having $\geq 30$ $\mathrm{kg} / \mathrm{m}^{2}$ BMI were considered as obese. The WC was measured at the midpoint between the lower border of the rib cage and the iliac crest. The biochemical parameters such as random blood sugar, serum glutamic oxaloacetic transaminase (SGOT), serum glutamic pyruvic transaminase (SGPT), SGOT:SGPT ratio, total bilirubin, indirect bilirubin, direct bilirubin, serum creatinine, blood urea, and alkaline phosphatases were estimated in all patients meeting the research requirements during the study visit using standardized methods.

\section{Ultrasonography imaging and examination}

A qualified radiologist examined the hepatic ultrasonic tests of all subjects on the same day. The radiologist categorized the levels of fatty liver into four as follows: Grade-0 "absence of steatosis with normal echogenicity of the liver"; Grade-1 "mild steatosis, echogenicity of the liver was greater than that of the right renal cortex, but the echogenic wall of the main portal vein was greater than that of the right renal cortex"; Grade-2 "moderate steatosis, impaired echogenicity of the main portal vein wall"; and Grade-3 "severe or impaired echogenicity of the main portal vein wall and impaired visualization of the posterior hepatic parenchyma or the diaphragm" (Dasarathy et al., 2009; Hernaez et al., 2011).

\section{Statistical analysis}

The continuous variables with normal distribution were expressed as mean and standard deviation. The risk of NAFLD was assessed using odds ratios and $95 \%$ confident intervals among the participants. Chi-square test was employed to estimate the differences in clinical characteristics in type-2 diabetes with or without NAFLD. The statistical analysis was carried out using Statistical Package for the Social Sciences 27.0 version.

\section{RESULTS}

Table 1 presents the comparison of mean and SD of patients with NAFLD and no NAFLD. Among the all parameters, the mean and SD of WC, random blood glucose (RBG) levels, and serum SGOT levels had a statistical significant association with patients with NAFLD, which indicates the probability of occurrence of NAFLD had a significant association $(p<0.05)$ with WC, RBG, triglycerides, and SGOT. The association between alcohol consumption and NAFLD is depicted in Table 2. The risk
Table 1. Characteristics of T2DM patients with or without NAFLD.

\begin{tabular}{lccc}
\hline \multirow{2}{*}{ Variables } & \multicolumn{2}{c}{ NAFLD } & \\
\cline { 2 - 3 } & $\begin{array}{c}\text { Yes }(\boldsymbol{n}=\mathbf{8 0}) \\
\text { Mean (SD) }\end{array}$ & $\begin{array}{c}\text { No }(\boldsymbol{n}=\mathbf{2 0}) \\
\text { Mean (SD) }\end{array}$ & \\
\hline Age (Years) & $40.68 \pm 12.2$ & $43.94 \pm 11.3$ & 0.303 \\
BMI (kg/m²) & $21.67 \pm 3.22$ & $21.86 \pm 1.15$ & 0.803 \\
WC (inches) & $35.72 \pm 2.47$ & $37.33 \pm 3.88$ & $0.028^{*}$ \\
Hemoglobin (g/dl) & $13.02 \pm 2.01$ & $12.81 \pm 2.09$ & 0.679 \\
Duration of diabetes (Years) & $4.40 \pm 2.08$ & $3.944 \pm 2.55$ & 0.422 \\
RBG (mg/dl) & $146.7 \pm 58.37$ & $119.5 \pm 36.84$ & $0.013^{*}$ \\
Triglycerides (mg/dl) & $176 \pm 34.5$ & $158 \pm 16.2$ & $0.025^{*}$ \\
SGOT/AST (mg/dl) & $50.48 \pm 43.2$ & $26.91 \pm 7.73$ & $0.023^{*}$ \\
SGPT/ALT (mg/dl) & $61.68 \pm 38.29$ & $46.48 \pm 25.98$ & 0.112 \\
SGOT:SGPT Ratio & $0.80 \pm 0.47$ & $0.69 \pm 0.25$ & 0.331 \\
Total bilirubin (mg/dl) & $2.40 \pm 2.63$ & $2.77 \pm 6.05$ & 0.682 \\
Direct bilirubin (mg/dl) & $1.03 \pm 1.13$ & $0.86 \pm 1.66$ & 0.601 \\
Indirect bilirubin (mg/dl) & $1.37 \pm 1.71$ & $1.89 \pm 4.49$ & 0.413 \\
Serum creatinine (mg/dl) & $0.88 \pm 0.25$ & $0.86 \pm 0.24$ & 0.691 \\
Blood urea nitrogen (mg/dl) & $23.05 \pm 5.40$ & $22.22 \pm 5.21$ & 0.558 \\
Alkaline phosphates (mg/dl) & $98.17 \pm 57.71$ & $89.11 \pm 35.41$ & 0.524 \\
\hline
\end{tabular}

${ }^{*} p$-value $<0.05$ was considered statistically significant.

of NAFLD is 6.65 times more among alcoholics and which showed statistical significance $(p<0.05)$, and remaining parameters were not statistically significant, However, male gender and patients with obesity had 1.72 times and 4.8 times chances of developing NAFLD, respectively, and it was comparatively 1.68 times more in non-graduates compared to graduates. This implies that the level of education has an impact on NAFLD. The risk of NAFLD is 2.66 time in both smokers and patients with less physical activity.

Table 3 shows the percentage proportions of fatty liver among the study participants. Grade-1 fatty liver was higher among male patients $(56,76.71 \%)$ compared to female patients $(17,62.96 \%)$. Among 150 patients, males are $12(16.44 \%)$ and females are $08(29.63 \%)$; the incidence of fatty liver was low in female subjects, which is statistically insignificant $(p<0.05)$. Table 4 illustrates the relationship between transaminases and severity of fatty liver disease. SGOT and SGPT are independent with our sample and are not related to the grade of fatty liver $(p<0.05)$.

\section{DISCUSSION}

Our study identified that the incidence of NAFLD among male subjects was $76.25 \%(n=61)$ and among female subjects was $23.75 \%(n=19)$. The results are nearly similar to Nigeria (68.8\%) (Afolabi et al., 2018). Asian countries, specifically south Asian countries, like India (30\%-90\%) and Sri Lanka (55\%) have a higher prevalence of NAFLD with T2DM (Amarapurkar et al., 2007; Farrell, 2003). The category of patients with NAFLD has significantly higher mean values of RBG levels, when compared to non-NAFLD patients. The uncontrolled glucose levels are positively linked with NAFLD $(p<0.05)$. WC is one of the common risk factors in NAFLD patients, which is strongly linked with NAFLD among our study subjects and similar to others. Our results support that individuals with NAFLD in the pre-diabetic stage and abnormal WC progress to the diabetic stage, which implies a positive association between NAFLD and diabetic onset (Lee et al., 2019). 
Table 2. The estimated odds of NFALD among T2 diabetic patients.

\begin{tabular}{|c|c|c|c|c|c|}
\hline \multirow{2}{*}{ Parameters } & \multicolumn{2}{|c|}{ NAFLD } & \multirow[b]{2}{*}{ OR } & \multirow[b]{2}{*}{$95 \% \mathrm{CI}$} & \multirow[b]{2}{*}{$p$ value } \\
\hline & Yes $(n=80 \%)$ & $\mathrm{No}(n=20 \%)$ & & & \\
\hline \multicolumn{6}{|l|}{ Gender } \\
\hline Male & $61(76.25)$ & $13(65.00)$ & 1.728 & $0.60-4.95$ & 0.30 \\
\hline Female $^{\mathrm{a}}$ & $19(23.75)$ & $07(35.00)$ & & & \\
\hline \multicolumn{6}{|l|}{ BMI $\left(\mathrm{kg} / \mathrm{m}^{2}\right)$} \\
\hline$\geq 25$ & $08(10.00)$ & $00(00.00)$ & 4.806 & $0.26-86.84$ & 0.28 \\
\hline$<25^{\mathrm{a}}$ & $72(90.00)$ & $20(100.00)$ & & & \\
\hline \multicolumn{6}{|l|}{ College graduate } \\
\hline Yes & $35(43.75)$ & $06(30.00)$ & 1.685 & $0.58-4.88$ & 0.33 \\
\hline $\mathrm{No}^{\mathrm{a}}$ & $45(56.25)$ & $13(70.00)$ & & & \\
\hline \multicolumn{6}{|l|}{ Physical activity } \\
\hline$\geq 3$ times per week & $32(40.00)$ & $04(20.00)$ & 2.666 & $0.81-8.70$ & 0.10 \\
\hline$<3$ times per week ${ }^{a}$ & $48(60.00)$ & $16(80.00)$ & & & \\
\hline \multicolumn{6}{|l|}{ Family history of DM } \\
\hline Yes & $27(33.75)$ & $10(50.00)$ & 0.509 & $0.18-1.37$ & 0.18 \\
\hline $\mathrm{No}^{\mathrm{a}}$ & $53(66.25)$ & $10(50.00)$ & & & \\
\hline \multicolumn{6}{|l|}{ Smoking } \\
\hline Yes & $04(05.00)$ & $00(00.00)$ & 2.774 & $0.14-53.72$ & 0.49 \\
\hline $\mathrm{No}^{\mathrm{a}}$ & $66(95.00)$ & $20(100.00)$ & & & \\
\hline \multicolumn{6}{|l|}{ Alcohol } \\
\hline Yes & $34(42.50)$ & $03(15.00)$ & 6.652 & $1.86-23.74$ & $0.00^{*}$ \\
\hline $\mathrm{No}^{\mathrm{a}}$ & $46(57.50)$ & $27(85.00)$ & & & \\
\hline \multicolumn{6}{|l|}{ Dyslipidemia } \\
\hline Yes & $48(60.00)$ & $13(70.00)$ & 0.923 & $0.37-2.47$ & 0.87 \\
\hline $\mathrm{No}^{\mathrm{a}}$ & $32(40.00)$ & $08(30.00)$ & & & \\
\hline
\end{tabular}

${ }^{\text {a}}$ Reference.

${ }^{*} p$-value $<0.05$ was considered statistically significant.

Table 3. The percentage proportions of fatty liver disease among both genders.

\begin{tabular}{lcccc}
\hline Grade of fatty liver & Male $(\boldsymbol{n}=\mathbf{7 3} \%)$ & Female $(\boldsymbol{n}=\mathbf{2 7} \%)$ & $\mathbf{9 5 \%} \mathbf{C I}$ & $\boldsymbol{p}$ value \\
\hline Normal liver & $12(16.44)$ & $08(29.63)$ & $-3.91-33.22$ & 0.14 \\
Grade-1 fatty liver & $56(76.71)$ & $17(62.96)$ & $-5.19-34.20$ & 0.17 \\
Grade-2 fatty liver & $04(05.47)$ & $02(07.41)$ & $-7.51-18.23$ & 0.79 \\
Grade-3 fatty liver & $01(01.37)$ & $00(00.00)$ & $-11.13-7.36$ & 0.54 \\
\hline
\end{tabular}

$p$-value $<0.05$ was considered statistically significant.

Table 4. Association of aminotransferases and severity of fatty liver.

\begin{tabular}{|c|c|c|c|c|c|}
\hline Grade (number) & $\begin{array}{c}\text { SGOT(>37U/I) } \\
\quad(n=48) \%\end{array}$ & $\begin{array}{c}\text { SGPT }(>43 \mathrm{U} / \mathrm{l}) \\
(n=33) \%\end{array}$ & $x^{2}, \mathrm{df}$ & $95 \% \mathrm{CI}$ & $p$ value \\
\hline Grade-1 (73) & $40(83.33)$ & $33(100)$ & $6.029,16.37$ & $3.54-29.58$ & $0.014^{*}$ \\
\hline Grade-2 (03) & $03(06.25)$ & $00(00)$ & 2.1156 .25 & $-4.95-16.83$ & 0.145 \\
\hline Grade-3 (01) & $01(02.10)$ & $00(00)$ & $0.693,2.10$ & $-8.46-10.92$ & 0.405 \\
\hline
\end{tabular}

In our study, the increased levels of Alanine aminotransferase (ALT) were likely to cause NAFLD than aminotransferase (AST) since ALT is a specific marker in hepatotoxicity and lipid accommodation than AST. The transaminases are generally elevated in NAFLD patients; in our study the probability of NAFLD was associated with SGOT, which is similar in Filipino-American women (Wong et al., 2008) and the incidence of grade-2 fatty liver was equivocal in either gender $(76.71 \%$ in men and $62.96 \%$ in women). Furthermore, there was a strong relationship (Table 4) between the elevation of SGOT and grade-1 fatty liver in our study participants ( $p=$ $0.014)$; SGOT levels were elevated in $48 / 100(48 \%)$ subjects and SGOT was elevated in 40/73 (54.80\%) grade-1 fatty liver patients. The grade of fatty liver is independent with regard to 
gender and is not statistically insignificant (Table 3). However, some previous studies have indicated that SGOT concentrations are not related well with hepatic steatosis severity and it may be common in high grades of steatosis (Chalasani et al., 2012; Spengler and Loomba, 2015). ALT levels were assessed in order to predict NAFLD in diabetic patients since it is the key marker in the accumulation of fat and liver injury.

Despite the above positive results, limitations still exist. For instance, NAFLD cases were diagnosed by conventional ultrasound apparatus, and this was not accompanied by advanced approaches, such as computerized tomography or any other high visualizing techniques. Additionally, based on the sample size, we cannot make a generalized conclusion from the results as the study contains only T2DM patients from one particular clinic.

\section{CONCLUSION}

NAFLD is commonly associated with tye- 2 diabetic patients and the incidence was high in our study site; however, this study denotes an alarming public health problem with increased $\mathrm{WC}$, poor control of blood glucose levels, and alcohol intake, which are major risk factors provoking NAFLD. Interventions such as health education, regular physical exercise, dietary modifications, regular health checkup, and proper medication adherence are recommended to lessen the risk of NAFLD in type2 diabetic patients.

\section{Future perspectives}

We will further assess the prognosis of fatty liver disease in type- 2 diabetics with NAFLD and without NAFLD by adopting the certain interventions.

\section{ACKNOWLEDGMENTS}

The authors are extremely thankful to study participants and the team of Bhargavi Gastro and Surgical Clinic for their comprehensive assistance and support to execute the research work.

\section{AUTHOR CONTRIBUTIONS}

All authors made substantial contributions to conception and design, acquisition of data, or analysis and interpretation of data; took part in drafting the article or revising it critically for important intellectual content; agreed to submit to the current journal; gave final approval of the version to be published; and agree to be accountable for all aspects of the work. All the authors are eligible to be an author as per the international committee of medical journal editors (ICMJE) requirements/guidelines.

\section{CONFLICT OF INTEREST}

The authors report no financial or any other conflicts of interest in this work.

\section{SOURCE OF FUNDING}

Self-funded study.

\section{COMPLIANCE WITH ETHICAL STANDARDS}

The study procedure was approved by the Institutional Ethics Committee, Dated: 10.12.2019 (IEC/19/Nov/71/10) of Sri Ramachandra Institute of Higher Education and Research
(Deemed to be University), Chennai, and has an affiliation with Bhargavi Gastro and Surgical Clinic Warangal, Telangana, India. This research was carried out in conjunction with good clinical practice guidelines.

\section{REFERENCES}

Afolabi BI, Ibitoye BO, Ikem RT, Omisore AD, Idowu BM, Soyoye DO. The relationship between glycaemic control and Non-alcoholic fatty liver disease in Nigerian type 2 diabetic patients. J Natl Med Assoc, 2018; 110(3):256-64.

Amarapurkar DN, Hashimoto E, Lesmana LA, Sollano JD, Chen PJ, Goh KL, Asia-Pacific working party on NAFLD1. How common is non-alcoholic fatty liver disease in the Asia-Pacific region and are there local differences? J Gastroenterol Hepatol, 2007; 22(6):788-93.

Chalasani N, Younossi Z, Lavine JE, Diehl AM, Brunt EM, Cusi $\mathrm{K}$, Charlton M, Sanyal AJ. The diagnosis and management of non-alcoholic fatty liver disease: practice guideline by the American Gastroenterological Association, American Association for the Study of Liver Diseases, and American College of Gastroenterology. Gastroenterology, 2012; 142(7):1592-609.

Dasarathy S, Dasarathy J, Khiyami A, Joseph R, Lopez R, McCullough AJ. Validity of real time ultrasound in the diagnosis of hepatic steatosis: a prospective study. J Hepatol, 2009; 51(6):1061-7.

Farrell GC. Non-alcoholic steatohepatitis: what is it, and why is it important in the Asia-Pacific region? J Gastroenterol Hepatol, 2003; 18(2):124-38

Forlani G, Giorda C, Manti R, Mazzella N, De Cosmo S, Ross MC, Nicolucci A, Di Bartolo P, Ceriello A, Guida P. The burden of NAFLD and its characteristics in a nationwide population with type 2 diabetes. $\mathrm{J}$ Diabetes Res, 2016; (1):1-9.

Goh GB, McCullough AJ. Natural history of nonalcoholic fatty liver disease. Dig Dis Sci, 2016; 61(5):1226-33.

Hernaez R, Lazo M, Bonekamp S, Kamel I, Brancati FL, Guallar E, Clark JM. Diagnostic accuracy and reliability of ultrasonography for the detection of fatty liver: a meta-analysis. Hepatology, 2011; 54(3):1082-90.

Kim D, Touros A, Kim WR. Nonalcoholic fatty liver disease and metabolic syndrome. Clin Liver Dis, 2018; 22(1):133-40.

Lee J, Cho YK, Kang YM, Kim HS, Jung CH, Kim HK, Park JY, Lee WJ. The impact of NAFLD and waist circumference changes on diabetes development in prediabetes subjects. Sci Rep, 2019; 9(1):1-8.

Li J, Zou B, Yeo YH, Feng Y, Xie X, Lee DH, Fujii H, Wu Y, Kam LY, Ji F, Li X. Prevalence, incidence, and outcome of non-alcoholic fatty liver disease in Asia, 1999-2019: a systematic review and metaanalysis. Lancet Gastroenterol Hepatol, 2019; 4(5):389-98.

Li Y, Wang J, Tang Y, Han X, Liu B, Hu H, Li X, Yang K, Yuan J, Miao X, Yao P. Bidirectional association between nonalcoholic fatty liver disease and type 2 diabetes in Chinese population: evidence from the Dongfeng-Tongji cohort study. PLoS One, 2017; 12(3):e0174291.

Mohan V, Farooq S, Deepa M, Ravikumar R, Pitchumoni C1. Prevalence of non-alcoholic fatty liver disease in urban south Indians in relation to different grades of glucose intolerance and metabolic syndrome. Diabetes Res Clin Pract, 2009; 84(1):84-91.

Singh SP, Nayak S, Swain M, Rout N, Mallik RN, Agrawal O, Meher C, Rao MV. Prevalence of nonalcoholic fatty liver disease in coastal eastern India: a preliminary ultrasonographic survey. Trop Gastroenterol, 2004; 25(2):76-9.

Spengler EK, Loomba R. Recommendations for diagnosis, referral for liver biopsy, and treatment of nonalcoholic fatty liver disease and nonalcoholic steatohepatitis. Mayo Clin Proc 2015; 90(9):1233-46.

Vernon G, Baranova A, Younossi ZM. Systematic review: the epidemiology and natural history of non-alcoholic fatty liver disease and non-alcoholic steatohepatitis in adults. Aliment Pharmacol Ther, 2011; 34(3):274-85. 
Wong CA, Araneta MR, Barrett-Connor E, Alcaraz J, Castañeda D, Macera C. Probable NAFLD, by ALT levels, and diabetes among Filipino-American Women. Diabetes research and clinical practice. 2008 Jan 1;79(1):133-40.

Younossi ZM, Golabi P, de Avila L, Paik JM, Srishord M, Fukui N, Qiu Y, Burns L, Afendy A, Nader F. The global epidemiology of NAFLD and NASH in patients with type 2 diabetes: a systematic review and metaanalysis. J Hepatol, 2019; 71(4):793-801.
How to cite this article:

Puneem US, Ramakrishnan SMR, Murthy SV, Nagasubramanian VR. Incidence of nonalcoholic fatty liver diseases and their associated risk factors among the type-2 diabetic population. $\mathrm{J}$ Appl Pharm Sci, 2021; 11(07):158-162. 